\title{
Structural Phase Transition Nomenclature Report of an IUCr Working Group on Phase Transition Nomenclature $\dagger$
}

\author{
J.-C. Tolédano, ${ }^{a} \ddagger$ A. M. Glazer,${ }^{b}$ Th. Hahn, ${ }^{c}$ E. Parthé, ${ }^{d, e}$ R. S. Roth,${ }^{f}$ R. S. Berry,,${ }^{g} \S$ R. MetselaAR ${ }^{h}$ And \\ S. C. ABrahams ${ }^{i * \dagger \dagger}$ \\ ${ }^{a}$ Laboratoire des Solides Irradiés, Ecole Polytechnique, F-91128 Palaiseau CEDEX, France, ${ }^{b}$ Clarendon Laboratory, \\ University of Oxford, Parks Road, Oxford OX1 3PU, England, ${ }^{c}$ Institut für Kristallographie, RWTH Aachen, \\ D-52056 Aachen, Germany, ${ }^{d}$ Department of Inorganic, Analytical and Applied Chemistry, University of Geneva, \\ 30 quai Ernest Ansermet, CH-1211 Geneva 4, Switzerland, ${ }^{e}$ Institute for Mineralogy and Crystallography, Geocenter \\ of the University of Vienna, A-1090 Vienna, Austria, ${ }^{f}$ B214 Materials Building, National Institute of Standards and \\ Technology, Washington, DC 20234, USA, ${ }^{g}$ Department of Chemistry, University of Chicago, 5735 South Ellis \\ Avenue, Chicago, IL 60637, USA, ${ }^{h}$ Laboratory for Solid State and Materials Chemistry, Eindhoven University of \\ Technology, PO Box 513, NL-5600 MB Eindhoven, The Netherlands, and ${ }^{i}$ Physics Department, Southern Oregon \\ University, Ashland, OR 97520,USA. E-mail: sca@mind.net
}

(Received 10 June 1998)

\begin{abstract}
A new nomenclature for the crystalline phases present in a sequence of structural phase transitions occurring as a function of temperature and/or pressure is defined as a first step in presenting a unified and informative notation for the various phases appearing in a phase diagram. This nomenclature specifies in a compact but intuitive manner the essential crystallographic and physical characteristics of each phase in the sequence. The current label in common use for certain phases $($ e.g $\alpha, I, \ldots)$, whenever such exists, is included in the new nomenclature. Eliminating confusion associated with the present multiple nomenclatures is expected to stimulate general use of the proposed new nomenclature. Examples of application to various types of solids are provided. Future extensions of the nomenclature are discussed.
\end{abstract}

\section{Introduction}

The International Union of Pure and Applied Chemistry (IUPAC) published its Definitions of Terms Relating to Phase Transitions in the Solid State (Clark et al.) in 1994,

\footnotetext{
$\dagger$ Established 15 February 1994 by the IUCr Commission on Crystallographic Nomenclature with all original members appointed 21 June 1994 under ground rules outlined in Acta Cryst. (1979), A35, 1072. The original Chairman P. Tolédano resigned from the Working Group 13 November 1995. J.-C. Tolédano was appointed Chairman 20 December 1995. R. L. Snyder resigned from the Working Group 28 April 1996 and R. S. Roth was appointed member 28 May 1996. The Report was received by the Commission 19 March 1998 and accepted 22 May 1998.

$\ddagger$ Chairman.

$\S$ Ex officio, International Union of Pure and Applied Physics.

- Ex officio, International Union of Pure and Applied Chemistry.

$\dagger+$ Ex officio, IUCr Commission on Crystallographic Nomenclature.

C) 1998 International Union of Crystallography

Printed in Great Britain - all rights reserved
}

with over 140 terms carefully elucidated. The document defines various types of transition including structural transitions of the solid state, metallurgical transitions, liquid-crystal transitions, solid-liquid or liquid-liquid transitions, transitions related to changes in composition, to changes in electronic structure and, in addition, describes many properties pertaining to these transitions. A systematic nomenclature for a sequence of several solid-state phase transitions was, however, not considered in the document. It is well known that such sequences occur almost as a rule in real materials and that the occurrence of a single transition only is relatively infrequent.

A multiplicity of terminologies may be found in the crystallographic and other literature of the condensed state for distinguishing individual members of a sequence of crystalline phases that form as a function of temperature and/or pressure. Common examples of notations are I, II, III; $\alpha, \beta, \gamma$; high, medium, low. Another widespread notation uses the point groups of the successive phases (e.g. $m \overline{3} m \rightarrow 4 m m \rightarrow 2 m m \rightarrow 3 m$ for $\mathrm{BaTiO}_{3}$ ). Confusion caused by the lack of a unified notation procedure led the Commission on Crystallographic Nomenclature to establish a Working Group on Phase Transition Nomenclature. The Working Group was charged with studying the multiple nomenclature in current use for naming the sequence of phases that a material may form as a function of temperature and/or pressure and with making such recommendations as may be appropriate. Researchers with experience in a broad range of systems undergoing solid-solid transitions were selected for membership in the Working Group, including representatives from IUPAC and the International Union of Pure and Applied Physics. ISSN 0108-7673 $\quad$ C) 1998 


\section{Basis for phase-transition nomenclature}

Discussion of the thermodynamic phase diagram of a substance has often been facilitated by use of a simple notation assigned to each phase in the diagram. Hence, the use of Greek letters $(\alpha, \beta, \ldots, \omega)$ is widespread in identifying the various phases in metals and alloys. The $\alpha$ and $\beta$ phases, for example, are commonly used to designate, respectively, the disordered (Cu-type) and the ordered (CsCl-type) phases of the $\mathrm{Cu}-\mathrm{Zn}$ alloy. Such notations are immediately understandable to workers familiar with the given metal or alloy since they refer to specific experimental conditions for observing these phases and to a definite set of experimental results (twinning appearance, mechanical properties etc.). However, this compact notation has several drawbacks. It is uninformative to the reader with only a general knowledge of the field of phase transitions, since it does not summarize any specific crystallographic or physical information. In addition, it is inflexible since the discovery of a new phase within a given diagram containing phases labelled $\alpha, \beta, \gamma, \ldots$ will usually require the introduction of more complicated symbols such as $\alpha^{\prime}$ or $\beta^{\prime}$. Further, it is not universal since different systems of labelling (e.g. I, II, ...) are common in fields other than metallurgy (e.g. that of structural transitions or the phase diagram of ice).

Our aim is to define a reasonably compact nomenclature for the phases that is both free from the preceding drawbacks and clearly preferable to the many current alternatives for the various disciplines dealing with crystalline phase transitions. In deciding the content of such a nomenclature, two types of questions have to be considered.

\subsection{Identification}

In the first place, a rapid and intuitive identification of each phase must be possible both for an expert on a specific substance and for other users. This implies a 'nickname' for each phase in the spirit of the labels currently used in the literature. Compatibility with existing notations requires the 'nickname' to incorporate these labels. On the other hand, the 'nickname' should also incorporate an element of intuitive identification for the general reader. We recommend that the range over which a given phase exists be used as an appropriate element of identification. In many cases, this is a temperature range. It may also be a pressure range or both thermal and pressure ranges.

The nickname of the high-temperature phase in quartz could hence be written $\beta|>846 \mathrm{~K}|$, while the tetragonal phase of barium titanate could be nicknamed II |403-278 K|. Such a convention has the advantage of flexibility. Consider for instance a phase nicknamed I $\left|T_{1}-T_{2}\right|$ (with $T_{1}>T_{2}$ ). The discovery of a new phase transition at $T_{3}$ between $T_{1}$ and $T_{2}$ would then lead to a sequence of two phases denoted I $\left|T_{1}-T_{3}\right|$ and $-\left|T_{3}-T_{2}\right|$ if the second phase has been assigned no label. Thus, this situation is dealt with by modifying the temperature range of the initially considered phase I. In systems that have been investigated for a long time, the most recently discovered phases may be recognized by the leading hyphen unless a label has been assigned by its discoverers.

\subsection{Informative character}

A fully informative description of the various phases formed by a given substance begins with the chemical formula and requires use of a large set of crystallographic and physical data, each part of which may be relevant to the expert. Hence, in addition to a knowledge of the structure type (e.g. perovskite) and space groups of the different phases (e.g. $P m \overline{3} m \rightarrow P 4 m m$ ), it may be necessary, in order to draw inferences concerning the properties of the substance, to have access to the relative positions of the various atoms in the unit cell of each phase, to their systematic change as a function of temperature across the transition, to the possibly singular behaviour of the anisotropic displacement ellipsoids of certain atoms etc. From the physical standpoint, a given phase may have many remarkable properties, e.g. magnetic, electric etc. Moreover, if ferroelectricity is present in a given phase, for instance, its bare mention is preferably extended by stating the magnitude of the spontaneous polarization, its temperature dependence and its structural origin (atomic displacement, electron cloud deformation, asymmetric occupation of certain sites, ...) etc.

Information of this kind, however, can be found readily in the published scientific reports on the given material; a sufficient nomenclature does not have to display all this information but only an appropriate fraction in simplified form. This format should lead the worker with a general knowledge of phase transitions to a straightforward identification of the transition category. The essential crystallographic information we recommend is provided by the space group and the number $(Z)$ of chemical formula units in the conventional unit cell, since these features are involved in a number of key aspects of phase transitions, as follows. Knowledge of the crystal symmetry on either side of a phase transition helps define the experimental methods that may be used to study the transition. A relationship of inclusion between the two space groups (i.e. if one is a subgroup of the other) of the phases surrounding a transition can suggest the possibility of a continuous transition (with zero latent heat). A change in point group across a transition indicates twinning with a definite pattern of variants. A change in the number of chemical formula units in the primitive (elementary) unit cell can denote the onset of a superstructure. In order to avoid possible ambiguity in the event of a change in unit-cell orientation from phase to phase, both 
space-group symbol and number, as given in International Tables for Crystallography (1996), are included. In compliance with normal crystallographic usage, we recommend giving the number of formula units in the conventional crystallographic (i.e. multiple if relevant) unit cell. Although physical information such as the appearance of a superstructure is clearly related directly to a primitive unit cell, derivation of one cell from another is a standard operation.

Secondly, the essential physical information retained in the new nomenclature is the ferroic character of the phase studied (i.e. ferromagnetic, ferroelectric, ferroelastic etc.). The main categories of ferroic materials have been defined in the IUPAC document (Clark et al., 1994) and elsewhere. This information allows inferences to be made regarding the twinning habit of the given phase and its response to external fields (magnetic, electric, mechanical stress etc.) and provides a guide to studying the corresponding phase transition.

Finally, it is recommended that certain specific information be added to the nomenclature in the form of a 'comment field', see also §3.6.

\section{Six-field phase-transition nomenclature}

The recommended nomenclature consists of a six-field notation for each phase with given chemical composition as follows, with field definitions summarized in $\S \S 3.1-3.6$. The fields are separated by vertical bars: the choice of experimental techniques best suited for

in $§ 4.9$. If more accurate limits are determined later, the earlier values should be replaced.

\subsection{Third field}

The space-group symbol and number of the phase, as used in International Tables for Crystallography (1996), should be given. When incomplete crystallographic information is available, these data may be replaced by specifying the point group $($ e.g. $4 \mathrm{~mm})$ or the crystal system (e.g. tetragonal).

\subsection{Fourth field}

The number of chemical formula units per conventional cell should be entered; if undetermined, the field should contain only a hyphen. The (tripled) hexagonal unit cell setting in rhombohedral systems should be used.

\subsection{Fifth field}

Contains the name of the ferroic property exhibited by the phase, see Clark et al. (1994). If this property has not actually been observed experimentally but is nevertheless probable on crystallographic grounds, a comment indicating this situation should be added in the sixth field. An unknown property is denoted by a hyphen (see $\$ 4.6$ for example).

\begin{tabular}{c|c|c|c|c|c}
$\begin{array}{c}\text { Usual label } \\
\text { in literature } \\
(\alpha, I, \ldots)\end{array}$ & $\begin{array}{c}\text { Temperature range (K), } \\
\text { pressure range }(\mathrm{Pa})\end{array}$ & $\begin{array}{c}\text { Space-group } \\
\text { symbol and No. }\end{array}$ & $\begin{array}{c}\text { Number of chemical } \\
\text { formula units per } \\
\text { conventional cell }\end{array}$ & $\begin{array}{c}\text { Ferroic } \\
\text { properties }\end{array}$ & Comments
\end{tabular}

\subsection{First field}

The label commonly associated with a given phase should be listed. If no label has been used in the literature for the phase, the field should contain a hyphen; nicknames may still be given to newly discovered phases. In the event that more than one label has been used, retention of the most common label is recommended.

\subsection{Second field}

If the phase is stable over a thermal range, then the temperature limits should be given in kelvins; if for a pressure range, in pascals. SI prefixes should be used as required. If no pressure range is indicated, the observations correspond to atmospheric pressure; similarly, if no temperature range is indicated, the observations correspond to room temperature. The more complex case of a pressure-temperature range is commented on

\subsection{Sixth field}

If the phase crystallizes with a standard structure type [for nomenclature and definitions of isotypism, see the IUCr Commission Report by Lima-de-Faria et al. (1990); for a handbook listing inorganic structure types, see TYPIX by Parthé et al. (1993, 1994); for a corresponding PC database, see TYPIX 1995 by Cenzual et al. (1995); and for a recent proposal that applies structuretype formulas to all inorganic materials, see Smith et al. (1998)], then the sixth field should begin with an entry in the format $\mid$ Type $=X X X X$. Typical structure-type names are, for instance, $X X X X=\mathrm{NaCl}$, pyrite, sphalerite etc., with the name in italics, or, alternatively, structure-type formulas such as $X X X X=G X X^{\prime}, T 3\left(T^{\prime} / L\right) X 4$ etc., with the formula in italics. The format is designed for future computer recognition, the italics indicating a standard structure-type name or formula and the period acting as a signal that further information in the field is unrelated to the structure type; if the name or formula is nonstandard or is not unique, this format should not be 
used. In the event the full six-field notation requires a terminating period (full stop) and is to be used within a longer sentence, the addition of delimiters around the notation such as square parentheses is recommended.

The remainder of the field is free form and may contain pertinent information such as transition characteristics, e.g. first order, Mott, martensitic, orderdisorder etc.; the number of variants corresponding to the ferroic orientations formed in the transition, not including antiphase domains related to the onset of superlattice reflections; additional information relative to incommensurability, if present; etc. Information in this field may be updated as necessary.

\section{Examples}

4.1. Barium titanate (BaTiO $)$ (Hewatt, 1974; Johnson, 1965) (see also Note added in proof)

$6 H|>1733 \mathrm{~K}| P 6_{3} / m m c$ (194) $\mid Z=6$ |non-ferroic |metastable at $300 \mathrm{~K}$ if cooled rapidly; stabilized by certain impurities.

I $|1733-403 \mathrm{~K} \quad| P m \overline{3} m$ (221) $\mid Z=1 \quad$ |non-ferroic |Type $=\mathrm{CaTiO}_{3}$ idealized. May exist up to melting if phase $6 H$ is not stabilized.

II $|403-278 \mathrm{~K} \quad| P 4 m m$ (99) $\mid Z=1 \quad$ |ferroelectric, ferroelastic $\mid 6$ ferroelectric, 3 ferroelastic variants.

III |278-183 K |Amm2 (38) $\mid Z=2$ |ferroelectric, ferroelastic $\mid 12$ ferroelectric, 6 ferroelastic variants.

IV $|<183 \mathrm{~K}| R 3 m$ (160) $\mid Z=3$ |ferroelectric, ferroelastic $\mid 8$ ferroelectric, 4 ferroelastic variants.

4.2. Potassium tellurium bromide $\left(K_{2} \mathrm{TeBr}_{6}\right)$ (Abrahams et al., 1984; Ihringer \& Abrahams, 1984)

I $|>434 \mathrm{~K} \quad| F m \overline{3} m \quad(225) \quad \mid Z=4 \quad$ |non-ferroic $\mid$ Type $=K_{2} P t C l$. Decomposes above $\sim 590 \mathrm{~K}$.

II $|434-400 \mathrm{~K}| P 4 / m n c(128) \mid Z=2$ |ferroelastic $\mid 3$ variants.

III $|<400 \mathrm{~K}| P 2_{1} / n(14) \mid Z=2$ |ferroelastic $\mid 12$ variants.

4.3. Lead aluminium fluoride $\left(\mathrm{Pb}_{5} A l_{3} F_{19}\right)$ (Andriamampianina et al., 1994; Sarraute et al., 1995, 1996; Omari et al., 1998)

I $|>670 \mathrm{~K}| \mathrm{I} / \mathrm{mcm}$ (140) $|Z=4|$ non-ferroic $\mid$ structure not yet unambiguously determined.

II $|670-360 \mathrm{~K}| I 4 / m$ (87) $|Z=4|$ ferroic* $\mid 2$ variants differing in elastic properties.

III $|360-320 \mathrm{~K}| I 2 / c(15)|Z=4|$ ferroelastic $\mid 4$ variants.

IV $|320-270 \mathrm{~K}| P 4 / n$ (85) $\mid Z=8$ |ferroic ${ }^{*}$ |antiferroelectric. *As in phase II.

$\mathrm{V}|<270 \mathrm{~K}| \mathrm{I} 4 \mathrm{~cm}$ (108) $|Z=4|$ ferroelectric $\mid 2$ variants.

4.4. Lead niobate $\left(\mathrm{PbNb}_{2} \mathrm{O}_{6}\right)$ (Roth, 1959, 1968; Labbé et al., 1977; Mahé, 1967)

I |>1423 K |tetragonal |- |nonferroic |metastable from 1423 to $873 \mathrm{~K}$ if cooled rapidly.
II $|<873 \mathrm{~K}| B b 2_{1} m$ (36) $|Z=40|$ ferroelectric |metastable at room temperature if cooled rapidly from $>1423 \mathrm{~K}$.

III $|<1423 \mathrm{~K}| R 3 m$ (160) $|Z=9|$ non-ferroic $\mid$ the stable phase at all temperatures below $1423 \mathrm{~K}$.

4.5. Tellurium dioxide $\left(\mathrm{TeO}_{2}\right.$ ) (Peercy \& Fritz, 1974; Worlton \& Beyerlein, 1975; Thomas, 1988)

$\alpha|<0.8 \mathrm{GPa}| P 4_{1} 2_{1} 2$ (92) $\mid Z=4$ |non-ferroic |room temperature structure determination.

$\beta \quad>0.8 \mathrm{GPa}\left|P 2_{1} 2_{1} 2 \quad(19) \quad\right| Z=4 \quad$ ferroelastic $\mid 2$ variants, room-temperature structure determined at $2 \mathrm{GPa}$.

4.6. Dicalcium silicate $\left(\mathrm{Ca}_{2} \mathrm{SiO}_{4}\right)$ (Eysel \& Hahn, 1970)

$\alpha|2400-1720 \mathrm{~K}| P 6_{3} m c$ (186) or $P 6_{3} / m m c$ (194) $\mid Z=2$ non ferroic $\mid$ Type $=\mathrm{K}_{2} \mathrm{SO}_{4}$ high. Stable.

$\alpha^{\prime}{ }_{-H}|1720-1430 \mathrm{~K}|$ Pcmn (62) $\mid Z=4$ |ferroelastic |Type $=\mathrm{K}_{2} \mathrm{SO}_{4}$ low. Stable.

$\alpha^{\prime}{ }_{L} \quad|1430-950 \mathrm{~K} \quad| C c m 2_{1}{ }^{*} \quad$ (36) $\quad|Z=16 \quad|-$ |Type $=\mathrm{K}_{2} \mathrm{SO}_{4}$ low, slightly deformed. Stable. *Other space groups are possible.

$\beta|<950 \mathrm{~K}| P 2_{1} / n$ (14) $|Z=4|-\mid$ Type $=\mathrm{K}_{2} \mathrm{SO}_{4}$ low, strongly deformed. Metastable with respect to $\gamma$.

$\gamma|<1000 \mathrm{~K}|$ Pcmn (62) $|Z=4|-\mid$ Type = olivine. Stable.

\subsection{Iron (Fe) (Donohue, 1974)}

$\delta|>1663 \mathrm{~K}| \operatorname{Im} \overline{3} m(229)|Z=2|$ non-ferroic $\mid$ Type $=W$. Melting at $1808 \mathrm{~K}$.

$\gamma|1663-1183 \mathrm{~K} \quad| F m \overline{3} m \quad$ (225) $\mid Z=4 \quad$ |non-ferroic |Type $=C u$.

$\beta|1183-1043 \mathrm{~K}| \operatorname{Im} \overline{3} m$ (229) $\mid Z=2$ |paramagnetic |Type $=W$.

$\alpha|<1043 \mathrm{~K}| \operatorname{Im} \overline{3} m \quad(229)^{*} \mid Z=2 \quad$ ferromagnetic $\mid$ Type $=W$. *Magnetic structure is pseudocubic.

$\varepsilon|>13 \mathrm{GPa}| P 6_{3} / m m c(194)|Z=2|-\mid$ Type $=M g$.

4.8. Yttrium (Y) (Grosshans et al., 1992)

- $|>46 \mathrm{GPa} \quad| F m \overline{3} m \quad$ (225) $\quad \mid Z=4 \quad$ non-ferroic |Type $=C u$.

- |46-26 GPa $\mid P 6_{3} / m m c$ (194) $\mid Z=4$ |non-ferroic |Type $=N d \alpha$.

- |26-12 GPa $|R \overline{3} m(166)| Z=9 \mid$ - |Type $=\operatorname{Sm} \alpha$.

- $\mid<12$ GPa $\left|P 6_{3} / m m c(194)\right| Z=2|-|$ Type $=M g$.

4.9. Caesium boron tetrafluoride $\left(\mathrm{CsBF}_{4}\right)$ (Richter \& Pistorius 1971)

This example is chosen to illustrate a situation in which the phase diagram is an intricate function of temperature and pressure. The proposed notation specifies a rectangular $(T, p)$ area for each phase, approximately superimposed on the actual range of the phase. Such a rough indication is generally insufficient to 
reconstruct, even approximately, the shape of the phase diagram. However, it provides the approximate stability range for the various phases and may be considered a first step in their identification. Only the three phases with identified crystal symmetries are listed below (labelled I, II, III in the published diagram) and only the first three fields of the nomenclature are entered:

I $|900-670 \mathrm{~K} ;<3 \mathrm{GPa}|$ cubic $\mid \ldots$

II $|670-500 \mathrm{~K} ;<0.8 \mathrm{GPa}|$ cubic $\mid \ldots$

III $|500-270 \mathrm{~K} ;<0.5 \mathrm{GPa}|$ orthorhombic $\mid \ldots$.

\section{Discussion}

The recommended nomenclature for each phase formed as the result of one or more structural phase transitions is the six-field notation described in $\$ 3$. However, following the first use in a paper of the full six-field notation for a given phase, it is recommended that, if the phase is commonly associated with a label, then the first two fields only be used later in the paper to identify that phase. If the phase is not commonly associated with a label, then the second two fields should be used.

Let us now consider possible extensions of this nomenclature. The mutually contradictory nomenclature systems currently used in the literature do not take into account a number of frequently encountered complex situations such as the formation of incommensurate phases. The new nomenclature can be readily and simply extended to such phases. In the third field, which normally specifies the space group, the space group of the average structure can instead be indicated while in the fourth field, which normally contains the number of formula units in the cell, the incommensurate character can be indicated as well as the number of independent modulation wave vectors, the direction(s) of modulation and the approximate modulation period in the appropriate crystallographic direction. The intermediate phase of quartz can hence be denoted:

- |846-844 K |P622 (177) |incomm.; $k=1$, a, 30a|- |-.

Within the framework of this supplementary convention, the succession of crystalline phases and an incommensurate phase in the case of potassium selenate $\left(\mathrm{K}_{2} \mathrm{SeO}_{4}\right)$, for example, can be denoted as follows:

$\mathrm{I}|>630 \mathrm{~K}| P 6_{3} / m m c(194)|Z=1|$ non-ferroic $\mid$-.

II $\mid 630-130 \mathrm{~K}$ |Pnam (62) $\mid Z=2$ |ferroelastic $\mid 3$ variants.

III |130-93 K |Pnam (62) |incomm.; $k=1$, a, $3 a$ ferroelastic $\mid 3$ variants.

IV $|<93 \mathrm{~K}|$ Pna2 $_{1}$ (33) $\mid Z=6$ |ferroelastic, ferroelectric $\mid 3$ ferroelastic variants; 6 ferroelectric variants.

Classifying certain long-period or polytype structures (such as $\mathrm{SiC}$ or $\mathrm{ZnS}$ ) as incommensurate, however, is not widely accepted by the scientific community. A similar difficulty concerns the occurrence of quasicrystalline phases which, possibly, could be specified by their orientational symmetry (point group). Consequently, extension of the new nomenclature to situations such as these, as well as to magnetic phase transitions, has been postponed to a later Report.

Another type of question that is presently unresolved concerns the possible inclusion, in the proposed nomenclature, of physical classifications such as the 'martensitic character' of a phase since these differ from the present ferroic classification. The difficulty in using both types of classification lies in the fact that, while the two concepts definitely overlap (martensitic and ferroelastic twinning is similar), their connection has not yet been fully clarified.

Note added in proof. Professor J. Kobayashi, Waseda University, has kindly pointed out that two new $\mathrm{BaTiO}_{3}$ phases have been reported (Sawaguchi et al., 1985, 1990; Akishige et al., 1990) to form on cooling the $6 H|>1733 \mathrm{~K}|$ phase. The first entry in $\$ 4.1$ should hence be replaced by the following three entries:

$6 H|>1733 \mathrm{~K}| P 6_{3} / m m c$ (194) $\mid Z=6$ |non-ferroic |metastable to $222 \mathrm{~K}$ if cooled rapidly from the stable phase above $1733 \mathrm{~K}$.

- $|222-74 \mathrm{~K}| C 222_{1}(20) \mid \quad Z=12$ |non-ferroic $\mid 6 H$ related.

- $|<74 \mathrm{~K}| P 2_{1}(4)|Z=6|$ ferroelectric, ferroelastic $\mid 6 H$ related.

It is a pleasure to thank Professor P. Tolédano, first Chairman of the Working Group for his guidance, and Professor G. Chapuis for serving as advisor to the Working Group.

\section{References}

Abrahams, S. C., Ihringer, J., Marsh, P. \& Nassau, K. (1984). J. Chem. Phys. 81, 2082-2087.

Akishige, Y., Oomi, G., Yamamoto, T. \& Sawaguchi, E. (1989). J. Phys. Soc. Jpn, 58, 930-939.

Andriamampianina, V., Gravereau, P., Ravez, J. \& Abrahams, S. C. (1994). Acta Cryst. B50, 135-141.

Cenzual, K., Gladyshevskii, R. \& Parthé, E. (1995). TYPIX 1995 Database of Inorganic Structure Types. Distributed by Gmelin-Institute for Inorganic Chemistry, Frankfurt, Germany.

Clark, J. B., Hastie, J. W., Kihlborg, L. H. E., Metselaar, R. \& Thackeray, M. M. (1994). Pure Appl. Chem. 66, 577-594.

Donohue, J. (1974). The Structure of the Elements. New York: John Wiley \& Sons.

Eysel, W. \& Hahn, Th. (1970). Z. Kristallogr. 131, 322-341.

Grosshans, W. A., Vohra, Y. K. \& Holzapfel, W. B. (1992). J. Magn. Mater. 29, 282-286.

Hewatt, A.W. (1974). Ferroelectrics, 6, 215-218.

Ihringer, J. \& Abrahams, S. C. (1984). Phys. Rev. B, 30, $6540-6548$.

International Tables for Crystallography (1996). Vol. A. Dordrecht: Kluwer Academic Publishers.

Johnson, C. J. (1965). Appl. Phys. Lett. 7, 221-224.

Labbé, Ph., Frey, M., Raveau, B. \& Monier, J. C. (1977). Acta Cryst. B33, 2201-2212.

Lima-de-Faria, J., Hellner, E., Liebau, F., Makovicky, E. \& Parthé, E. (1990). Acta Cryst. A46, 1-11. 
Mahé, R. (1967). Bull. Soc. Chim. Fr. pp. 1879-1884.

Omari, M. E., Réau, J. M., Sénégas, J., Ravez, J., Abrahams, S. C., Nadiri, A. \& Yacoubi, A. (1998). J. Chem. Phys. 108, 2896-2902.

Parthé, E., Gelato, L., Chabot, B., Penzo, M., Cenzual, K. \& Gladyshevskii, R. (1993, 1994). TYPIX. Standardized Data and Crystal Chemical Characterization of Inorganic Structure Types. In Gmelin Handbook of Inorganic and Organometallic Chemistry, four volumes, 1596 pp. Berlin: Springer. (Types found with oxides and halides are included only in specific cases).

Peercy, P. S. \& Fritz, I. J. (1974). Phys. Rev. Lett. 32, 466-470. Richter, P. W. \& Pistorius, C. W. F. T. (1971). J. Solid State Chem. 3, 197-199.

Roth, R. (1959). J. Res. Natl Bur. Stand. (US), 62, 27-38.
Roth, R. (1968). J. Res. Natl Bur. Stand. (US), 72A, 175-186. Sarraute, S., Ravez, J., Bravic, G., Chasseau, D. \& Abrahams, S. C. (1995). Acta Cryst. C51, 1731-1732.

Sarraute, S., Von der Mühll, R., Bravic, G., Chasseau, D., Feigelson, R. S. \& Abrahams, S. C. (1996). Acta Cryst. B52, 72-77.

Sawaguchi, E., Akishige, Y. \& Yamamoto, T. (1985). J. Phys. Soc. Jpn, 54, 480-482.

Sawaguchi, E., Akishige, Y. \& Yamamoto, T. (1990). Ferroelectrics, 106, 63-68.

Smith, D. M., Roberts, A. C., Bayliss, P. \& Liebau, F. (1998). Am. Mineral. 83, 126-132.

Thomas, P. A. (1988). J. Phys. C, 21, 4611-4627.

Worlton, T. G. \& Beyerlein, R. A. (1975). Phys. Rev. B, 12, 1899-1907. 